\title{
Mechanical characterization and modeling for anodes and cathodes in lithium-ion batteries
}

\author{
Lubing Wang ${ }^{\mathrm{a}, \mathrm{b}}$, Sha Yin ${ }^{\mathrm{a}, \mathrm{b}}$, Chao Zhang ${ }^{\mathrm{c}, \mathrm{d}}$, Yong Huan ${ }^{\mathrm{e}}$, Jun $\mathrm{Xu}^{\mathrm{f}, \mathrm{g}, *}$ \\ a Department of Automotive Engineering, School of Transportation Science and Engineering, Beihang University, Beijing, 100191, China \\ ${ }^{\mathrm{b}}$ Advanced Vehicle Research Center, Beihang University, Beijing, 100191, China \\ ${ }^{\mathrm{c}}$ Department of Aeronautical Structure Engineering, Northwestern Polytechnical University, Xi'an, Shaanxi 710072, China \\ d Joint International Research Laboratory of Impact Dynamics and Its Engineering Application, Northwestern Polytechnical University, Xi'an, Shaanxi 710072, China \\ e State Key Laboratory of Nonlinear Mechanics (LNM), Institute of Mechanics, Chinese Academy of Sciences, Beijing, 100190, China \\ ${ }^{\mathbf{f}}$ Department of Mechanical Engineering and Engineering Science, The University of North Carolina at Charlotte, Charlotte, NC 28223, United States \\ ${ }^{\mathrm{g}}$ North Carolina Motorsports and Automotive Research Center, The University of North Carolina at Charlotte, Charlotte, NC 28223, United States
}

\section{H I G H L I G H T S}

- Electrode behaviors were characterized by mechanical-electrochemical factors.

- Mechanical behaviors of electrodes are strain rate, electrolyte and SOC dependent.

- Electrochemical status influences mechanical behaviors of electrodes greatly.

- Constitutive models for electrodes are established for future computation modeling.

\section{A R T I C L E I N F O}

\section{Keywords:}

Battery electrodes

Mechanical integrity

Battery safety

Strain rate effect

SOC dependency

\section{G R A P H I C A L A B S T R A C T}

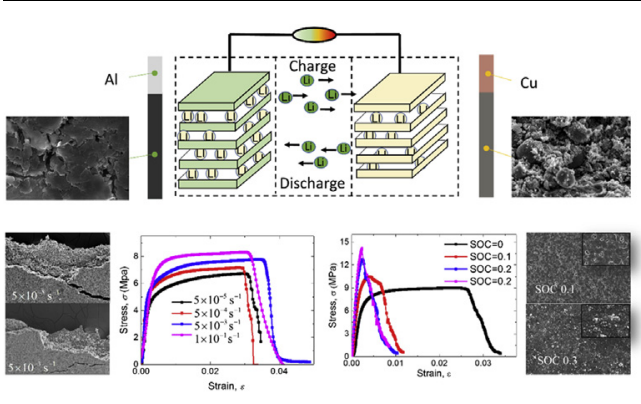

\begin{abstract}
A B S T R A C T
Mechanical properties of electrode materials have significant influence over electrochemical properties as well as mechanical integrity of lithium-ion battery cells. Here, anode and cathode in a commercially available 18650 NCA (Nickel Cobalt Aluminum Oxide)/graphite cell were comprehensively studied by tensile tests considering material anisotropy, SOC (state of charge), strain rate and electrolyte content. Results showed that the mechanical properties of both electrodes were highly dependent on strain rate and electrolyte content; however, anode was SOC dependent while cathode was not. Besides, coupled effects of strain rate and SOC of anodes were also discussed. SEM (scanning electron microscope) images of surfaces and cross-sections of electrodes showed the fracture morphology. In addition, mechanical behavior of $\mathrm{Cu}$ foil separated from anode with different SOC values were studied and compared. Finally, constitutive models of electrodes considering both strain rate and anisotropy effects were established. This study reveals the relationship between electrochemical dependent mechanical behavior of the electrodes. The established mechanical models of electrodes can be applied to the numerical computation of battery cells. Results are essential to predict the mechanical responses as well as the deformation of battery cell under various loading conditions, facilitating safer battery design and manufacturing.
\end{abstract}

\footnotetext{
* Corresponding author. Department of Mechanical Engineering and Engineering Science, The University of North Carolina at Charlotte, Charlotte, NC 28223, United States.

E-mail address: jun.xu@uncc.edu (J. Xu).
} 


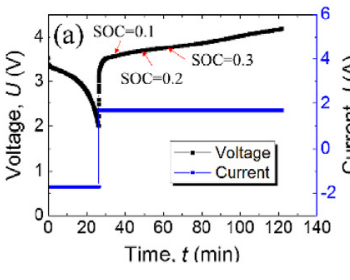

(c)

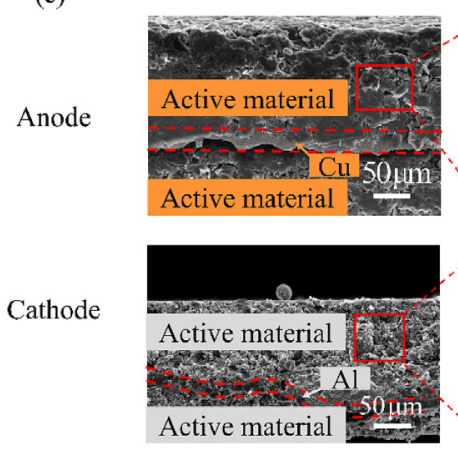

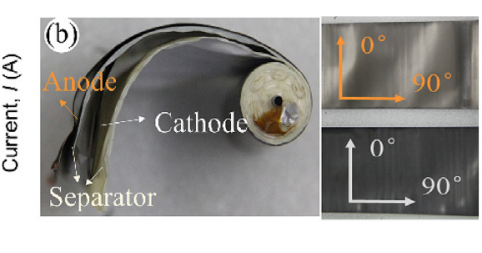

Cross-section
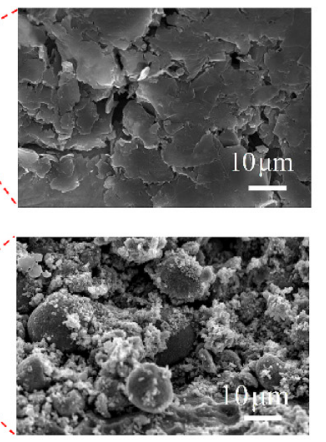

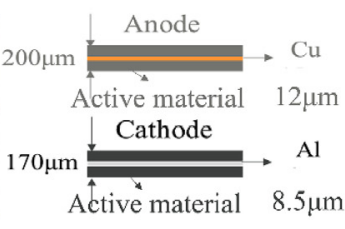

Surface
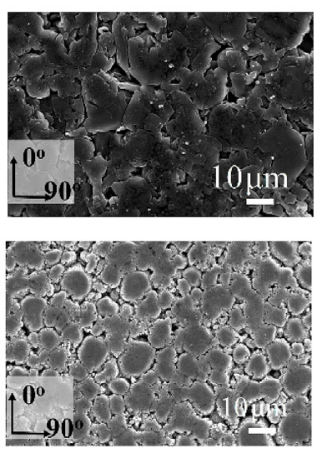

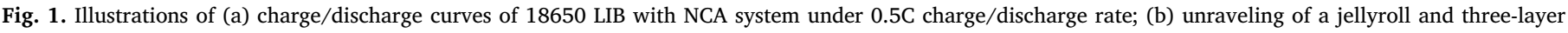
composite structure of anode and cathode; and (c) The micro morphologies of anode and cathode from cross-section and surface views.

\section{Introduction}

Safety issues of lithium-ion batteries (LIBs) have been bottlenecks in further application of LIBs in electric vehicles [1-4]. The internal components (i.e. the separator, anode and cathode) would fail or fracture once the battery cell suffers from mechanical abusive loadings, triggering the irreversible internal short-circuit [5-7]. Then a large amount of heat is generated during the internal short-circuit and serious chemical decomposition reactions are ignited, followed by a great quantity of venting gas. This is referred to as the thermal runaway process, which causes the internal pressure to increase rapidly, eventually leading to fire or explosion [8-10]. Therefore, a full understanding of loading-dependent and electrochemical-dependent mechanical behaviors of lithium-ion battery components is essential and urgent in battery safe design, optimization and evaluation.

Pioneering studies on mechanical behavior of lithium-ion battery components were conducted from both micro- and macro-length scales. From micro-length level, stress evolution, distribution and fracture analysis of particles on electrode current collectors with the diffusion of lithium ions during charging and discharging were investigated [11-14]. The expansion of cathode/anode particles would cause grain fracture and thus lead to battery capacity deterioration or sudden failure $[15,16]$. Also, the charge/discharge would cause the exfoliation and failure of SEI membrane [17] as well as the adhesive failure due to electrode volume change $[18,19]$. In the meantime, volume change of electrodes due to intercalation/de-intercalation can also introduce extra stress within the battery [20]. From macroscale perspective, the mechanical properties of different types of separators, such as single layer [21] and composite polymer layers [22,23], were studied in respects of strain rate [24-26], temperature [24], solvent [27,28] and anisotropy [29] based on the mechanical experiments and numerical simulations. Xiao et al. [30] conducted creep test and frequency sweep, discovered the anisotropy of the separator. Arnold et al. [28] studied the in-situ mechanical behavior of polymer separators in solvent environment and found that the mechanical properties of separator would be weaken by solvent. Further, Xu et al. [31] performed comprehensive mechanical tests of both two types of separators, considering both the anisotropy and environment solvent effects coupled with the effect of strain rate. In the meantime, the in-plane tensile properties [32,33] and out-plane compression performances [34] of electrodes were studied with LIBs in $\mathrm{LiCoO}_{2} / \mathrm{LixC}_{6}$ system. Sahraei et al. [33] found that the active material particle would influence the mechanical properties of current collector. In addition, the SOC dependency of anode on out-plane compression properties was first discovered by $\mathrm{Xu}$ et al. [34]. Recently, Ma et al. [35] established an electrochemical-irradiated plasticity model of metallic electrodes and studied their yield strengths affected by SOC. Besides, the constitutive models were developed to describe the stressstrain response of electrodes, the most simple and general form is $\sigma=E \varepsilon$ (where $\sigma$ is stress matrix, $E$ is elastic constants matrix and $\varepsilon$ is strain matrix), and the failure criterion is also established [36].

Since LIB cells with NCA cathodes are widely applied in electric vehicles due to high energy and power density, however, little is known about the mechanical behaviors of LIB cell and its component materials. To bridge this critical gap, in this paper, mechanical behavior of electrodes in the NCA cells is comprehensively investigated in terms of mechanical loading conditions (i.e. anisotropicity and strain rate dependency) and electrochemical status of the cells (i.e. SOC and solvent dependency). Furthermore, simple and effective constitutive models for both electrodes are established, facilitating further mechanical analysis and numerical simulation of LIB study.

\section{Experimental}

\subsection{Materials}

Material samples were taken out from fresh (discharged to cut-off voltage then charged to a specific SOC value) 18650 lithium-ion batteries based on a $\mathrm{Li}_{\mathrm{x}}\left(\mathrm{Ni}_{0.80} \mathrm{Co}_{0.15} \mathrm{Al}_{0.05}\right) \mathrm{O}_{2}$ (NCA) cathode, whose charging and discharging characteristic curves are shown in Fig. 1a with the SOC values noted. The jellyroll of the lithium-ion battery shown in Fig. 1b was rolled in separator-cathode-separator-anode sequence. The electrode is a current foil coated with the particulate coating on both sides with thickness of $200 \mu \mathrm{m}$ and $170 \mu \mathrm{m}$ in anode and cathode, respectively. Specifically, the anode was $12 \mu \mathrm{m} \mathrm{Cu}$ foil coating with $\mathrm{Li}_{0.008} \mathrm{C}_{6}$ while the cathode was $8.5 \mu \mathrm{m} \mathrm{Al}$ foil coating with $\mathrm{Li}_{0.925}\left(\mathrm{Ni}_{0.80} \mathrm{Co}_{0.15} \mathrm{Al}_{0.05}\right) \mathrm{O}_{2}$. Here, the axial and rolling directions of battery are defined as $0^{\circ}$ and $90^{\circ}$, respectively. Electrodes samples were prepared as square shapes for the following tensile tests, and one layer of electrode was tested in each tensile test.

Scanning electron microscope (JSM 6010L) was also employed to observe the microstructure of two electrodes, results are shown in Fig. 1c. Three-layer composite structure was observed for both anode 

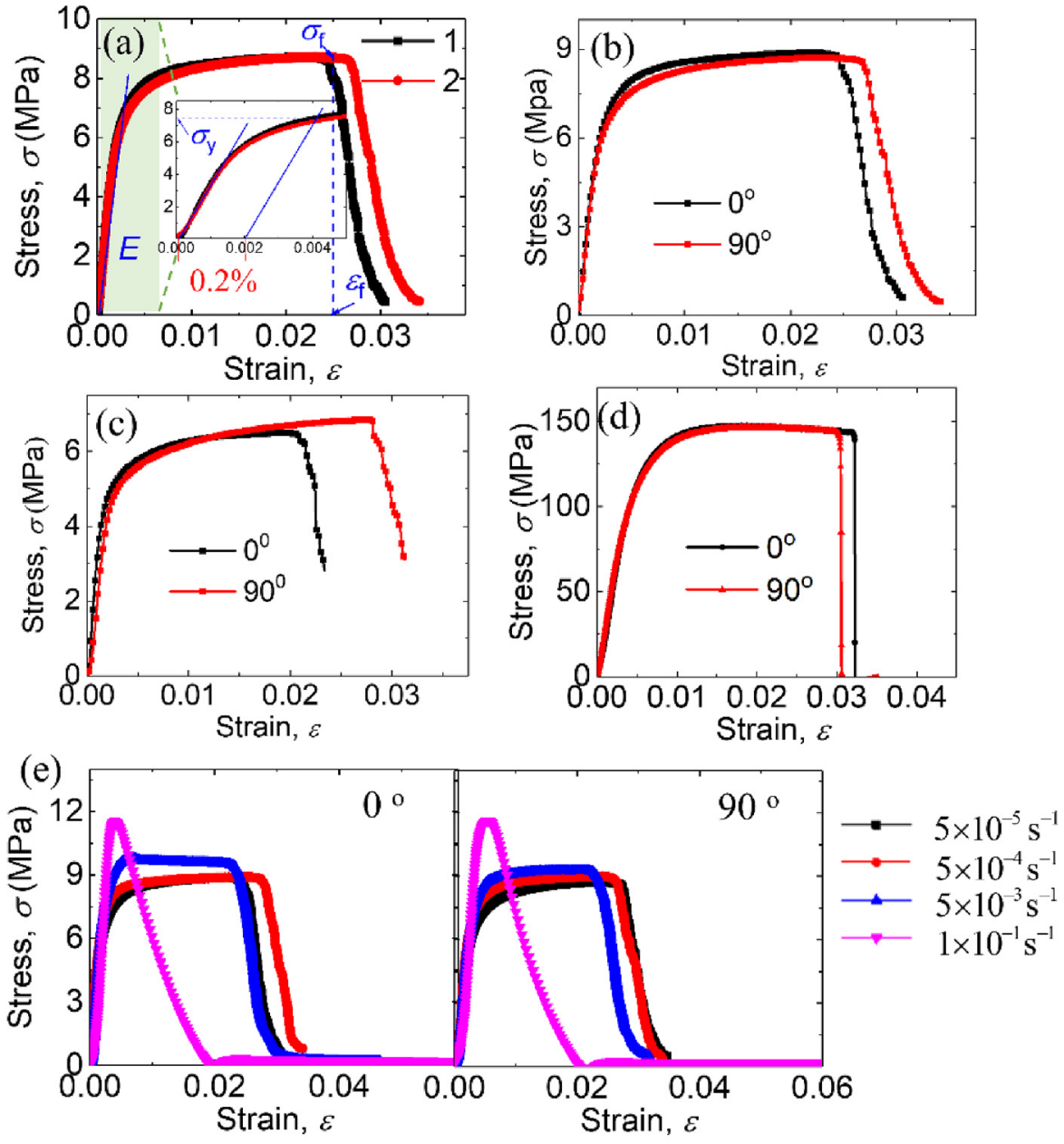

(f)

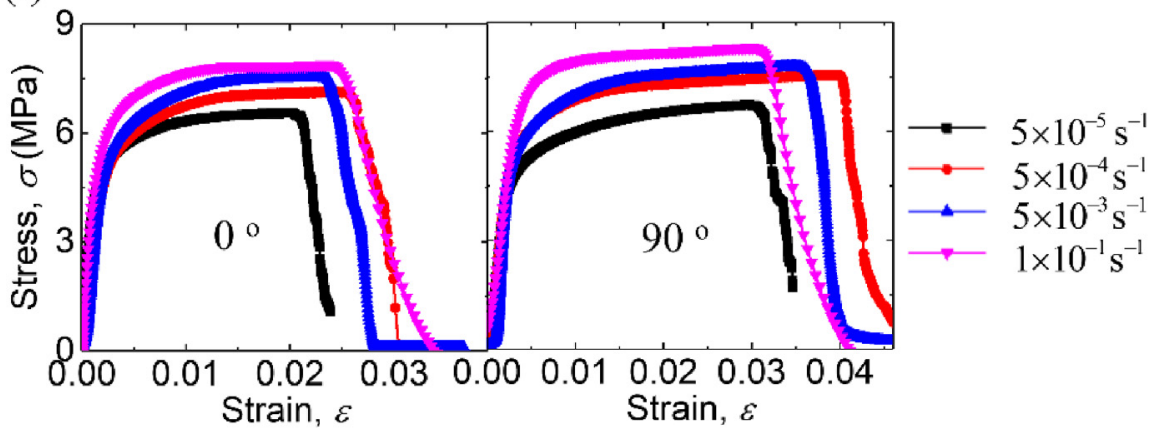

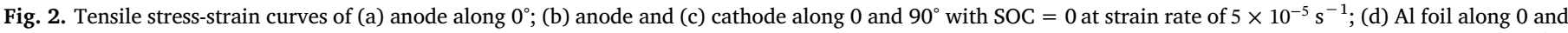
$90^{\circ}$. Representative tensile stress-strain curves of (e) anode along 0 and $90^{\circ}$; (f) cathode along 0 and $90^{\circ}$ at different strain rates varying from $5 \times 10^{-5}$ to $0.1 \mathrm{~s}^{-1}$.

and cathode materials, and the active material of anode is much denser than that of cathode. For cathode materials, the morphology of their active materials were spherical particles while that of anode materials had no obvious profile among active particles. Also, the surface morphology for the electrode materials showed obvious traces of compaction from rolling process.

\subsection{Methods}

A series of tensile tests were designed by considering effects of strain rate, material anisotropy, SOC dependency and electrolyte effect. Samples at different strain rates ranging from $5 \times 10^{-5} \mathrm{~s}^{-1}$ to $0.1 \mathrm{~s}^{-1}$ along $0^{\circ}$ and $90^{\circ}$ directions were tested to explore the coupled effect of material anisotropy and strain rate. Besides, SOC dependency and electrolyte effect were also studied at $5 \times 10^{-4} \mathrm{~s}^{-1}$. Instron E1000 was employed to investigate mechanical behavior of anode and cathode with a load capacity of $200 \mathrm{~N}$ and accuracy of $0.01 \mathrm{~N}$. Each experiment was repeated at least three times to ensure the credibility of the data.

\section{Results}

The stress-strain curves of electrode materials were shown in Fig. 2 at a specific strain rate of $5 \times 10^{-5} \mathrm{~s}^{-1}$ with $\mathrm{SOC}=0$. As shown in Fig. $2 \mathrm{a}$ and $\mathrm{b}$, the typical tensile stress of anode and cathode along $0^{\circ}$ direction increases linearly with strain followed with a long plastic yield stress plateau until failure, indicating ideal elastic-plastic behavior similar to metal. The slope of the curve is defined as the effective modulus $E$ and yield stress $\sigma_{\mathrm{y}}$ is the stress at which $0.2 \%$ plastic 

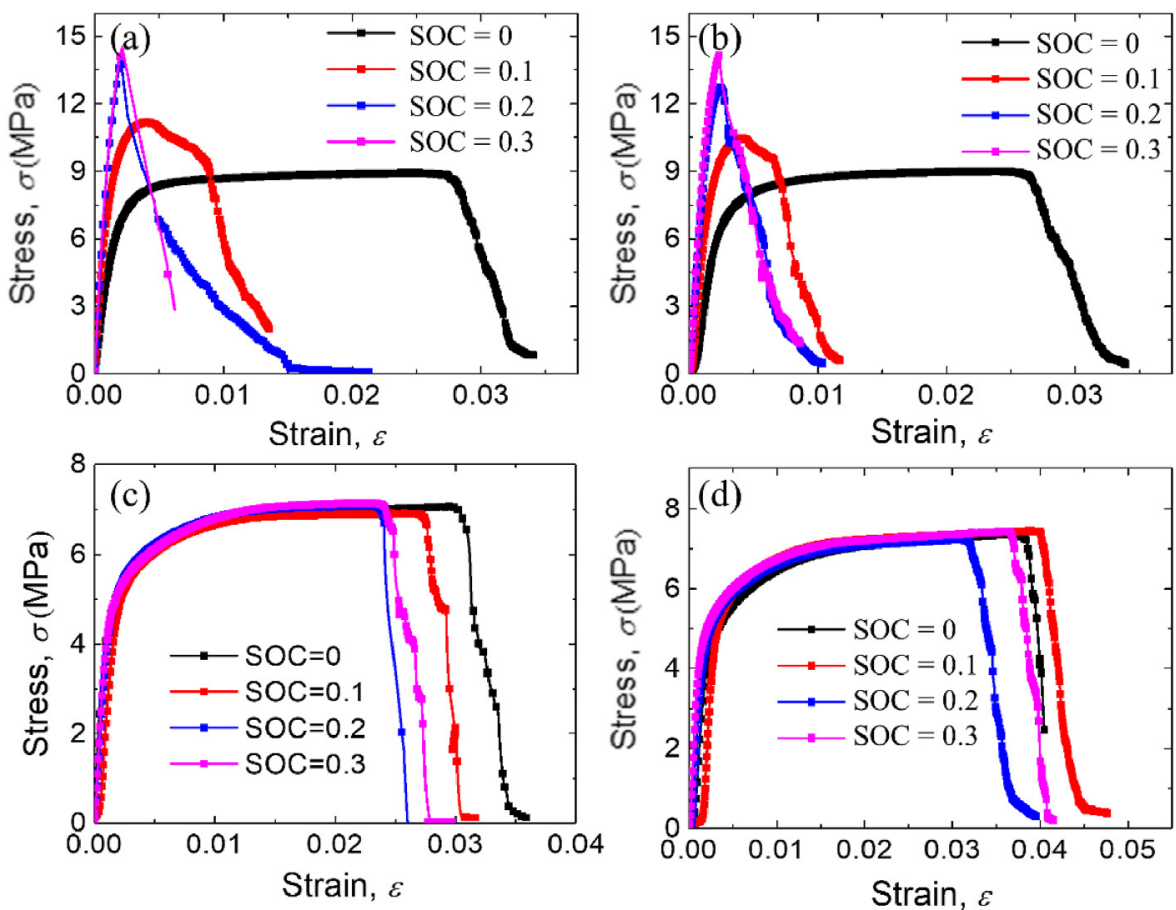

(e)

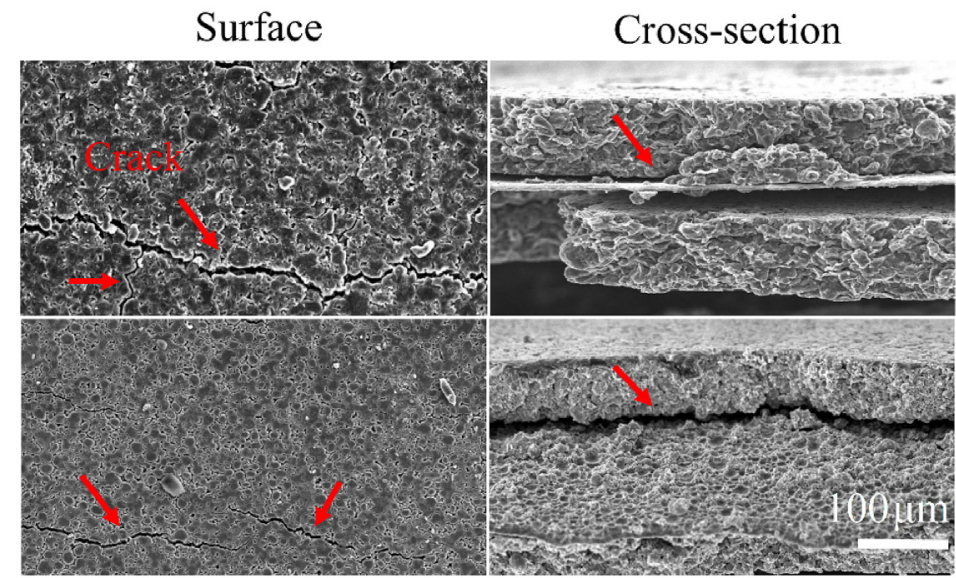

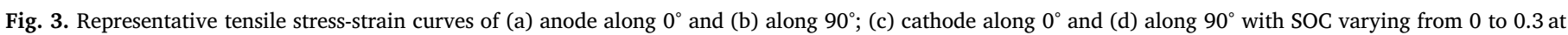
strain rate of $5 \times 10^{-4} \mathrm{~s}^{-1}$. SEM images of fracture surface for anode and cathode from surface and cross-section views.

deformation occurs shown in the subplot of Fig. 2a. Meanwhile, the failure stress $\sigma_{\mathrm{f}}$ and strain $\varepsilon_{\mathrm{f}}$ are defined as the onset of failure. Tensile responses of anode along $0^{\circ}$ and $90^{\circ}$ directions were compared in Fig. $2 \mathrm{~b}$ with no difference observed, and the effective modulus $E=3.64 \mathrm{GPa}, \sigma_{\mathrm{y}}=7.7 \mathrm{MPa}, \sigma_{\mathrm{f}}=8.3 \mathrm{MPa}$ and $\varepsilon_{\mathrm{f}}=0.026$. As for cathode, the effective modulus and failure strain were different along different directions indicating strong anisotropic behavior as shown in Fig. 2c, i.e. effective moduli along $0^{\circ}$ and $90^{\circ}$ directions are $E_{0}=3.62 \mathrm{GPa}$ and $E_{90}=2.50 \mathrm{GPa}$; while failure strain along $0^{\circ}$ and $90^{\circ}$ directions are 0.02 and 0.03 . To explain the anisotropy behavior, the commercial aluminum foil (component of cathode) was tested in tension and the resultant stress-strain curves were shown in Fig. 2d which is isotropic along different directions. We attribute cathode anisotropy to the bending stress induced by its assembly process for aluminum foil is softer than copper foil. Note that the yield and failure stress are almost the same, i.e. $\sigma_{\mathrm{y}}=5.5 \mathrm{MPa}$ and $\sigma_{\mathrm{f}}=6.4 \mathrm{MPa}$.

Fig. 2e-f shows the representative stress-strain curves at different strain rates varying from $5 \times 10^{-5}$ to $0.1 \mathrm{~s}^{-1}$. As illustrated, strain rate effect is obvious on electrodes in two material directions. All the curves exhibit strain rate "hardening" phenomenon, leading to greater yield and failure strength values. Interestingly, at the strain rate of $0.1 \mathrm{~s}^{-1}$, anode materials show brittle behavior with no yield in both 0 and $90^{\circ}$ as shown in Fig. 2e. As for cathode, all the curves behaved similarly at various strain rates without ductile-brittle transition observed as shown in Fig. 2f. As a composite structure, the deformation incoordination of anode is more obvious because particles of anode were more readily to peel off than cathode as strain rate increases, and thus the corresponding failure mode transformed while failure strain decreased.

The tensile responses of anode and cathode at different SOC values were shown in Fig. 3a-d. Anode is highly dependent of SOC (Fig. 3a and b) while cathode is not (Fig. 3c and d). For anode, the yield and failure strength increase while the failure strain decreases with SOC. Specifically, as $\mathrm{SOC}=0$, the anode can be termed as ideal elastic-plastic material while as SOC $=0.1$, the stress-strain curve can be divided into four parts including elastic, plastic, softening and failure phases. The corresponding $\sigma_{\mathrm{y}}$ and $\sigma_{\mathrm{f}}$ at $\mathrm{SOC}=0.1$ are $10.8 \mathrm{MPa}$ and $9 \mathrm{MPa}$ respectively, which are both greater than those at $\mathrm{SOC}=0$; while $\varepsilon_{\mathrm{f}}=0.01$, is $67 \%$ smaller than that in SOC $=0$. Additionally, when $\mathrm{SOC} \geq 0.2$, the anode material behaved as elastic-brittle material, and the strength of which is still larger with the SOC. Interestingly, the decrease rate of the failure strain has the same trend with the increase rate of strength (maximum stress), both decrease with the SOC, and reach the highest 
(a)
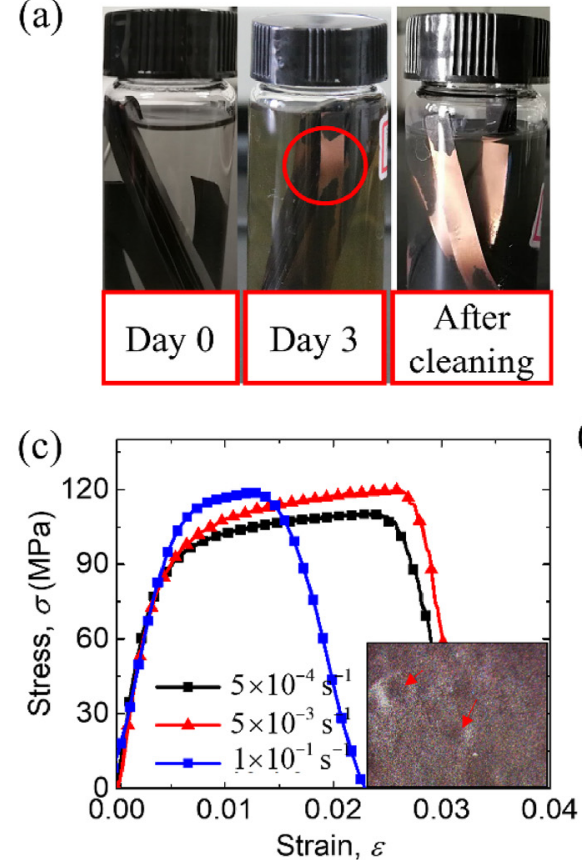

(e)

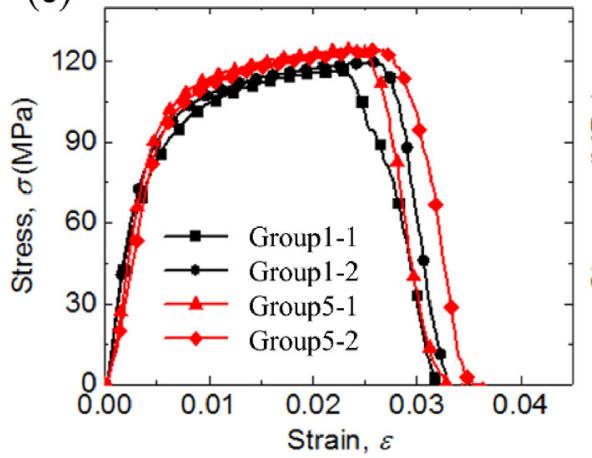

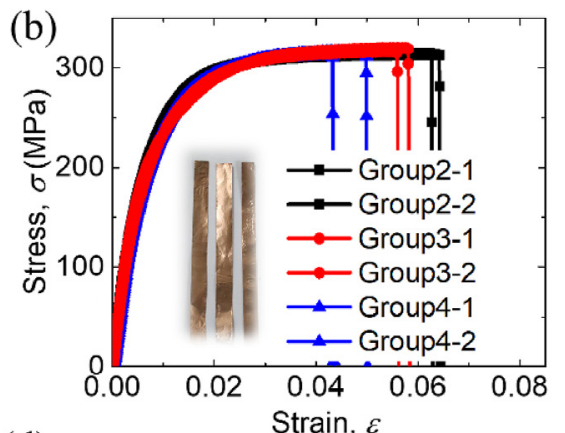

(d)

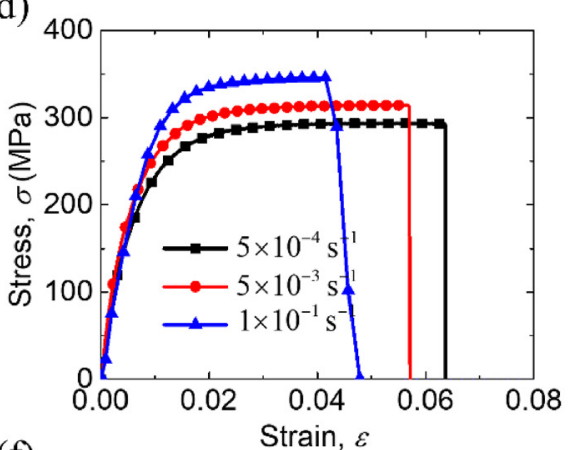

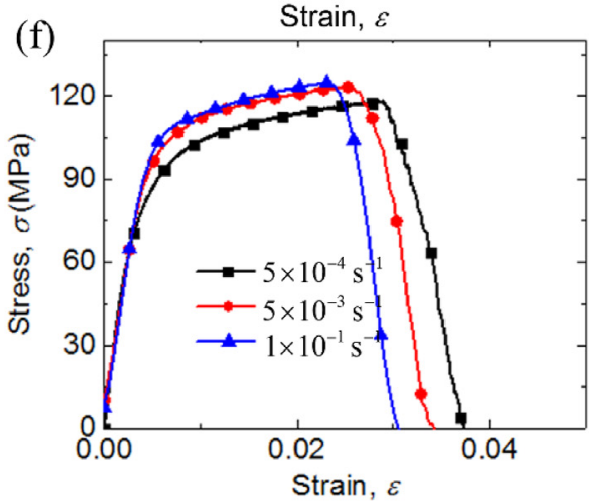

Fig. 4. (a) Preparation of all the Cu foils by soaking in methanol; stress-strain curves of samples from (b) Groups 2,3 and 4 at $5 \times 10^{-3} \mathrm{~s}^{-1}$; (c) Group 1 and (d) Group 2 at different strain rates; (e) Groups 1 and 5 at $5 \times 10^{-3} \mathrm{~s}^{-1}$; (f) Group $5(\mathrm{SOC}=0.3)$ at different strain rates. Note that Group 1: Cu foils separated from anodes with SOC 0; Group 2: commercially available Cu foils; Group 3: commercial $\mathrm{Cu}$ foils were methanol soaked only; Group 4: commercial Cu foils were ultrasound cleaned only; Group 5: Cu foils obtained from anodes with SOC 0.3.

value when SOC changes from 0 to 0.1 (Fig. 3a-b). As Xu et al. [34] discovered that the battery cell was SOC dependent with SOC various from 0 to 0.7 which mainly caused by anode material, we infer that anode material will also show the SOC dependent with higher SOC values. For cathode, SOC has little influence on its mechanical properties, and the failure strain along $90^{\circ}$ is larger than that along $0^{\circ}$ just as mentioned before.

\section{Discussion}

\subsection{Effects of manufacturing and Li-ion intercalation}

As discussed in Section 2, the electrode materials were composed of current collector and active material, while $\mathrm{Cu}$ current collector for anode and $\mathrm{Al}$ for cathode. Failure mechanisms were investigated through SEM images of anode and cathode after tensile tests as shown in Fig. 3e. Transverse and longitudinal cracks were observed on the surface of electrodes nearby the fracture section, indicating earlier failure of active material than current collector. From the image taking from the anode fracture cross-section, interfacial debonding happened between active particles and current collector. Thus, the deformation incoordination was readily to happen which also supported the failure mode transition phenomenon as strain rate increased in Section 3 for anode. As for cathode, the fracture occurred through the active particle layer while the interface kept intact.

To further understand effects of manufacturing and Li-ion intercalation on mechanical properties variation of electrodes, Cu current collector for anodes were studied as a typical example. Subsequently, $\mathrm{Cu}$ foils without active material obtained from four distinguished ways were testing and compared. Firstly, the anode was firstly soaked in methanol for three days (Fig. 4a) and cleaned in an ultrasound cleaner for four minutes under $40 \mathrm{kHz}$ frequency, named as Group 1. Also, commercially available $\mathrm{Cu}$ foil without any further processing, and that soaked in methanol or ultrasound cleaning were used for comparison defined as Groups 2, 3 and 4, respectively. The tensile stress-strain curves of anode from groups 1-4 were shown in Fig. 4b-d. Methanol soaking and ultrasound cleaning have no influence in the effective modulus of $\mathrm{Cu}$ foil but smaller failure strain from Fig. 4b for Groups 2-4. However, significant difference was observed comparing with Groups 1 and 2 from Fig. $4 \mathrm{c}$ and d, and the effective moduli of $\mathrm{Cu}$ foil were $30 \mathrm{GPa}$ and $50 \mathrm{GPa}$. Also, the failure strength and strain of Group 1 at strain rate of $5 \times 10^{-3}$ decreased by $47.8 \%$ and $66.7 \%$ compared with those of Group 2, respectively. We attributed the decrease to the pits induced by manufacturing process.

$\mathrm{Li}$-ion can penetrate $\mathrm{Cu}$ grain boundaries during charging/discharging [37]. So as to illustrate its influence, $\mathrm{Cu}$ foils obtained with the 

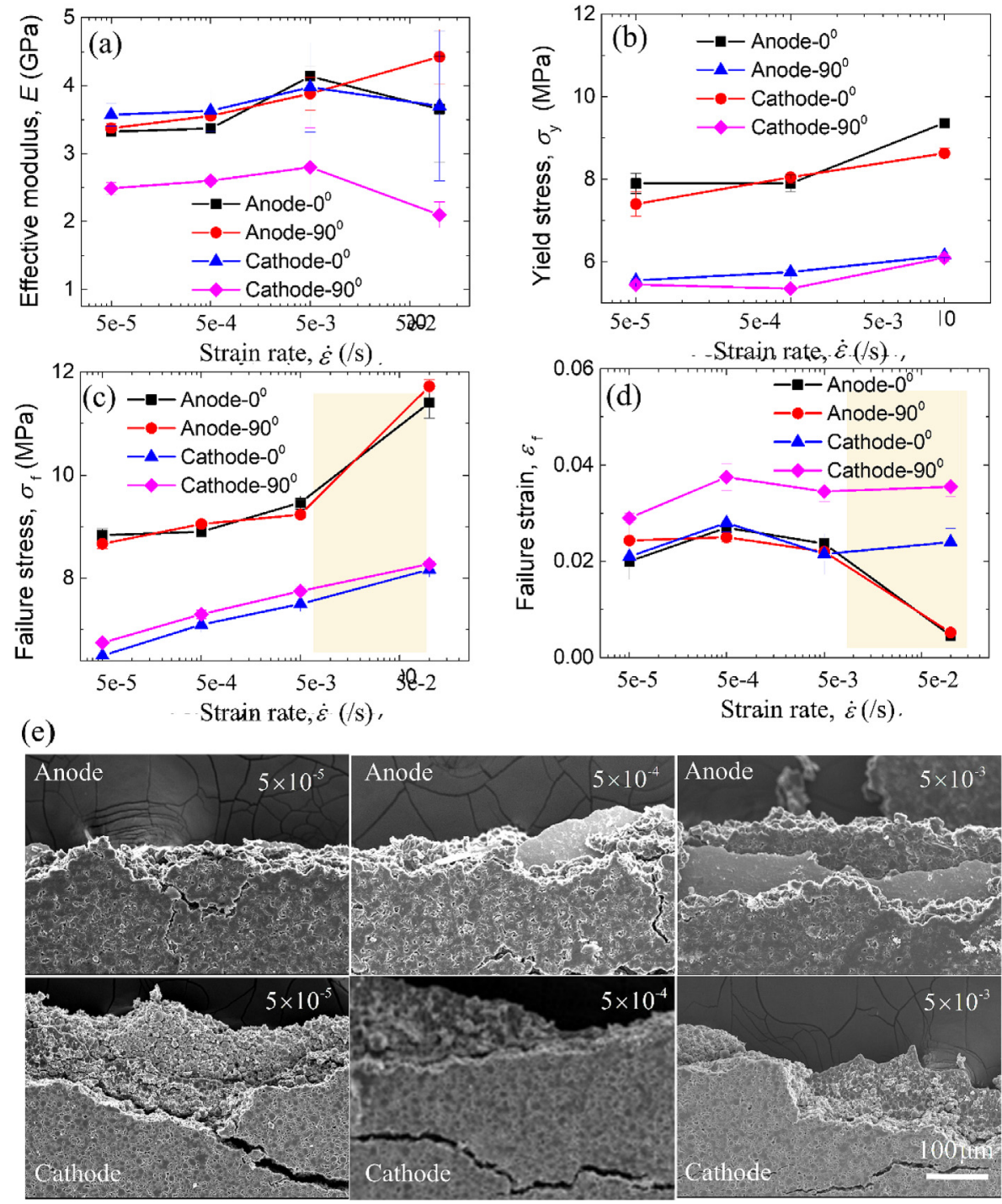

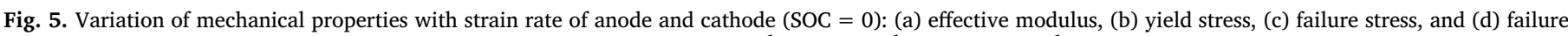
strain. (e) the fracture surface SEM figures of anode and cathode at $5 \times 10^{-5} \mathrm{~s}^{-1}, 5 \times 10^{-4} \mathrm{~s}^{-1}$ and $5 \times 10^{-3} \mathrm{~s}^{-1}$, respectively.

same method as Group 1 from anodes with SOC 0.3, named as Group 5, were tested and compared with Group 1. As shown in Fig. 4e, the failure stress of $\mathrm{Cu}$ foils in Group 5 was 5\% greater than that in Group 1, indicating strengthening effects may be caused by Li-ion intercalation. Note that the strain rate effect at higher SOC value was also observed here as shown in Fig. 4f. Note that even if the mechanical properties of the anode and $\mathrm{Cu}$ foils are obtained, it is still difficult to infer the mechanical behavior of the active materials due to the complex interface and the binders effect.

\subsection{Strain rate effect and SOC dependency}

Fig. 5a-d were the summary for governing mechanical properties, i.e. $E, \sigma_{\mathrm{y}}, \sigma_{\mathrm{f}}$ and $\varepsilon_{\mathrm{f}}$, curves of which with error bars at different strain rates and material directions of electrodes were built up. For both anode and cathode materials, $E$ and $\sigma_{\mathrm{y}}$ increase gently with strain rate, as shown in Fig. 5a and b. As for $\sigma_{\mathrm{f}}$ in Fig. $5 \mathrm{c}$, a nearly liner increase with strain rate was observed for both cathode and anode, until a sharp increase appeared for anode as strain rate reached up to $0.1 \mathrm{~s}^{-1}$. Correspondingly, the variation of failure strain $\varepsilon_{\mathrm{f}}$ with strain rate was shown in Fig. $5 \mathrm{~d}$ and $\varepsilon_{\mathrm{f}}$ of anode decreased sharply at $\dot{\varepsilon}=0.1 \mathrm{~s}^{-1}$. The failure strain effected by strain rate could also be explained via SEM images shown in Fig. 5e, from which the deformation incoordination of anode with strain rate was obviously detected while little difference for cathode.

To better investigate SOC effect of electrodes, quantitative relations with error bars about typical mechanical properties under different SOC values of electrodes were shown in Fig. 6a-d. Cathode is SOC independent from the blue and magenta lines in these four figures with the corresponding SEM images (Fig. 6e) about cathode in various SOC values with little difference. Because Lithium-ions de-intercalates from $\mathrm{Li}_{0.925}\left(\mathrm{Ni}_{0.80} \mathrm{Co}_{0.15} \mathrm{Al}_{0.05}\right) \mathrm{O}_{2}$ mainly through a valence state change rather than crystallographic structure leading to little influence over mechanical properties [38,39]. As for anode, the mechanical stiffness and strength varied with SOC specially from 0.1 to 0.2 illustrated in the yellow shaded region (Fig. 6a and c), and the failure strain $\varepsilon_{\mathrm{f}}$ decreased with SOC. Also, Fig. 6e showed the microcosmic surface of anode with SOC from 0.1 to 0.3 , and the granular layer is much denser as SOC increased. Lithium-ions intercalated into anode during charging [40,41] and the corresponding stiffness and strength increased [34], because the guest atoms diffusing into a host solid would cause the host too brittle to flow under a mechanical load [42].

Further, coupled effects of SOC and strain rate were also studied. Anode samples with SOC $=0.3$ were studied as a typical example and tested along $0^{\circ}$ under different strain rates. The tensile stress-strain curves were shown in Fig. 7a. Comparing with those for SOC $=0$, strain 

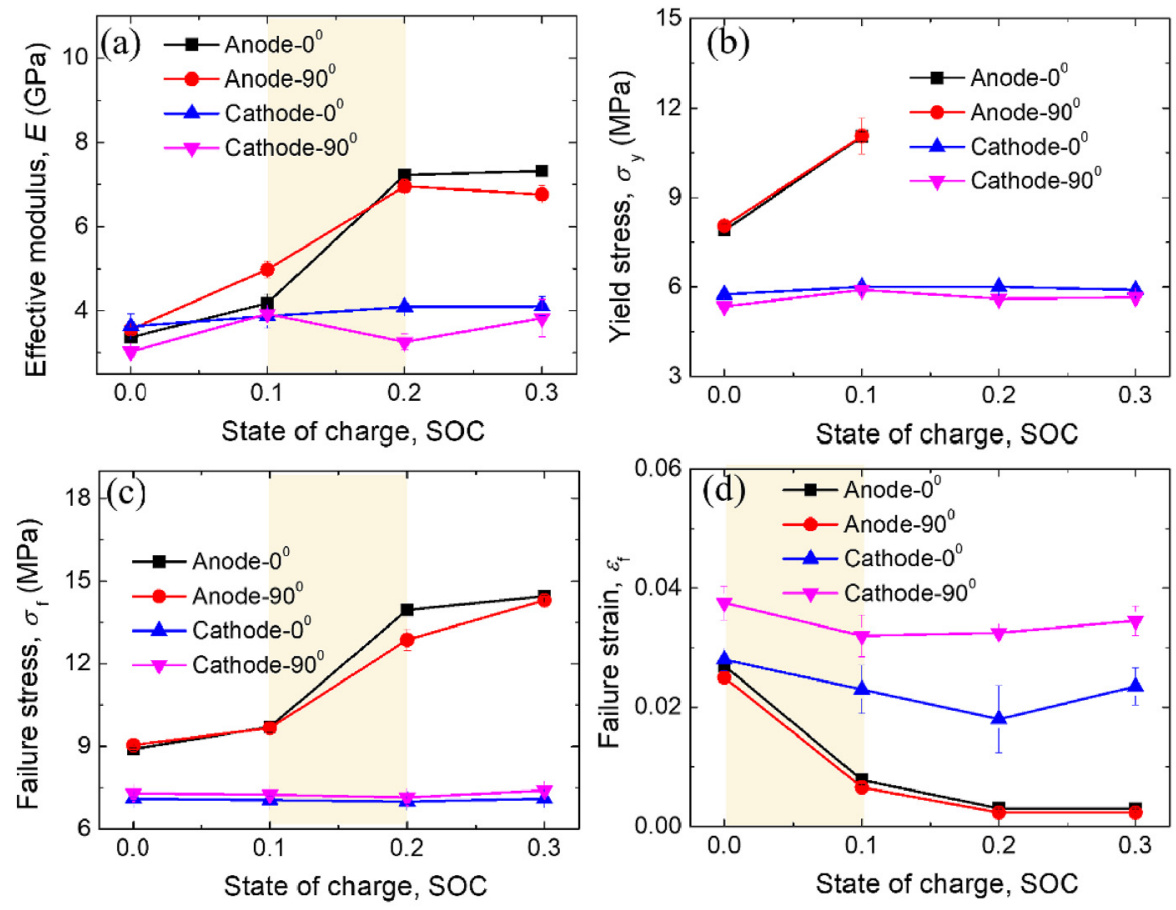

(e)

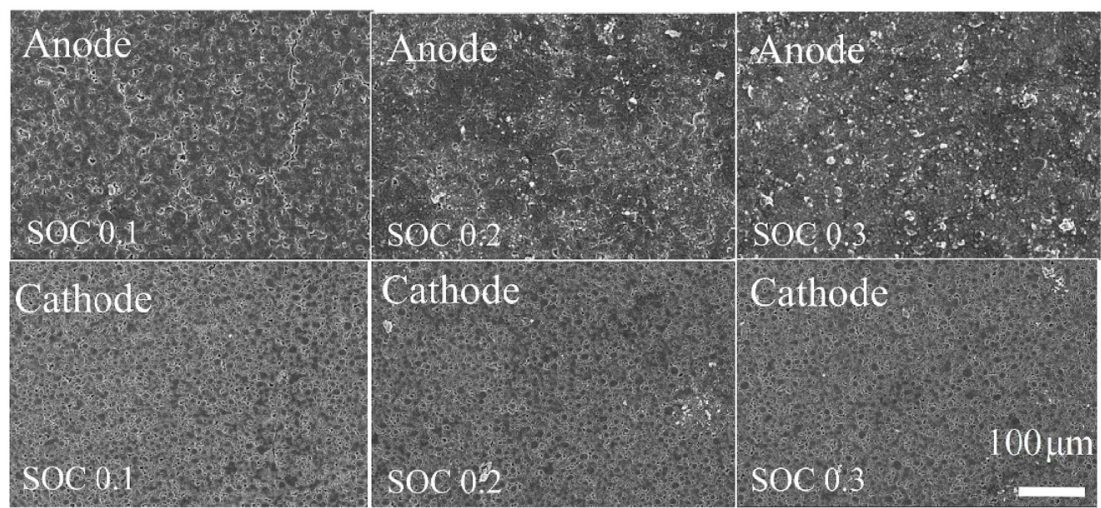

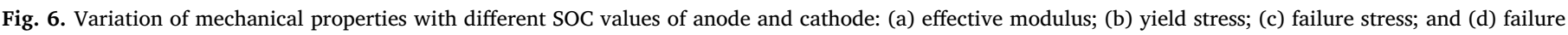
strain. (e) SEM images for anode and cathode with $S O C=0.1,0.2,0.3$, respectively.

rate dependency was also observed for samples with $\mathrm{SOC}=0.3$. The strength increases monotonously with strain rates varying from $5 \times 10^{-5}$ to $5 \times 10^{-3} \mathrm{~s}^{-1}$ but decreases as the strain rate increased up to $0.1 \mathrm{~s}^{-1}$ as shown in Fig. 7b. For SOC $=0.3$, more lithium-ions would intercalate into the anode, and thus decreased the interfacial adhesive force. The active material peeled off from $\mathrm{Cu}$ foil at strain rates varying from $5 \times 10^{-5}$ to $5 \times 10^{-3} \mathrm{~s}^{-1}$ as shown in Fig. $7 \mathrm{a}$-11, which lead to higher strength. Samples need to further overcome the friction between active materials and $\mathrm{Cu}$ foil during the tensile tests at relatively low strain rate, which made the stress increment higher than that at strain rate of $0.1 \mathrm{~s}^{-1}$ when the active material has no time to peel off (as shown in (2).

\subsection{Electrolyte implication}

To investigate electrolyte effect, two groups of samples with different electrolyte conditions were prepared: one was made immediately after battery disassembling and the other seven days after disassembling. All the electrode samples were tested and the results were shown in Fig. 7c and d. Samples without electrolyte had greater modulus and strength than those with electrolyte, which meant that electrolyte weakened the mechanical properties of anode and cathode. The penetration of electrolyte would cause reduction of local densities and thus softer mechanical properties; meanwhile, it helped the active material and current collector deform more coordinately which resulted in greater failure strain values. The influence of electrolyte on cathode is much more obvious than that on anode: $85.9 \%$ and $445 \%$ increase in failure strain, $8 \%$ and $12.7 \%$ drop in strength of anode and cathode respectively with electrolyte. As such, cathode is observed to be more sensitive to electrolyte environment.

\subsection{Mechanical constitutive model}

Mechanical constitutive model is important and essential in mechanical integrity analysis for LIB. As discussed, anode can be regarded as isotropic material, and thus the ideal elastic-plastic model is adopted to describe its mechanical properties. Rambery-Osgood model is used to describe its elastic-plastic behavior which can be expressed as

$\varepsilon=\frac{\sigma}{E}+0.016\left(\frac{\sigma}{9}\right)^{18.77}$

where $E=3640 \mathrm{MPa}$, and the model prediction is shown in Fig. 8a with a good agreement with typical experiment data at strain rate of $5 \times 10^{-5}$. To further optimize the model, the strain rate effect is included, and the expression can be modified as 

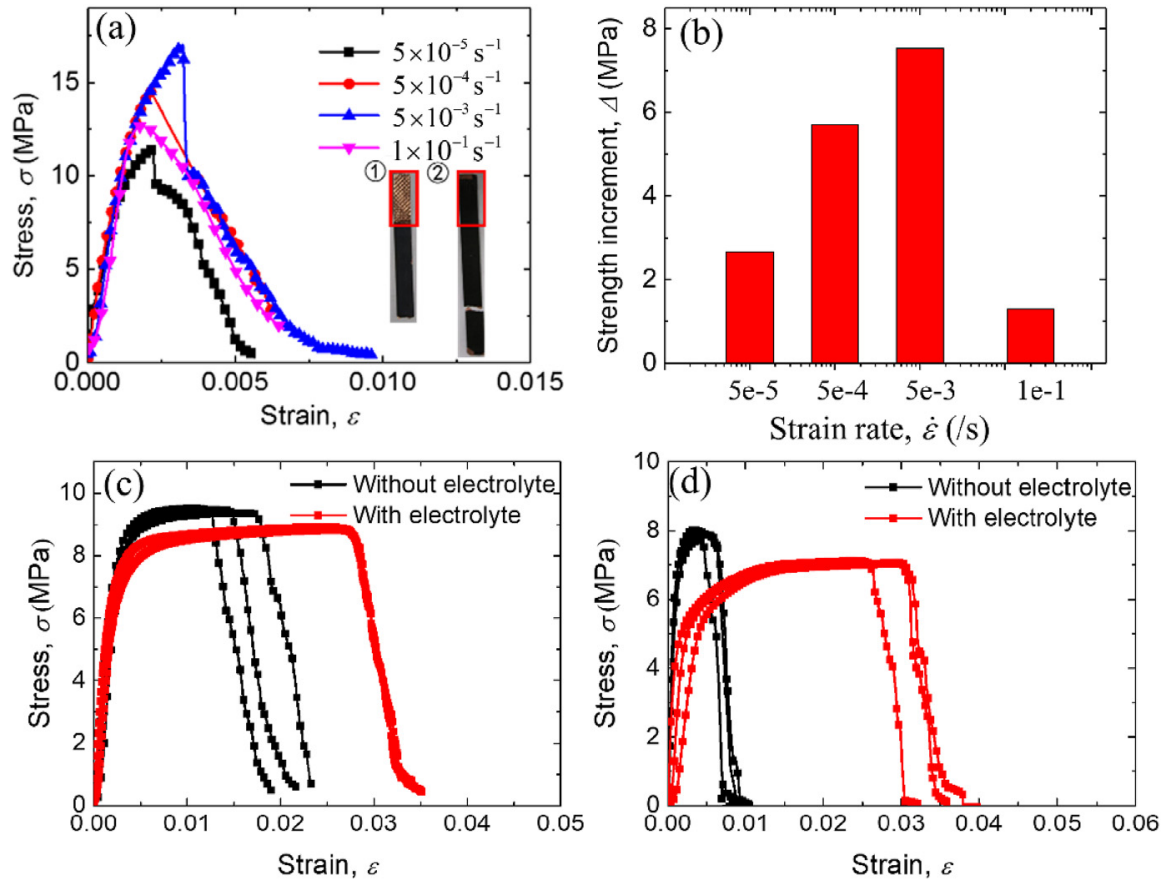

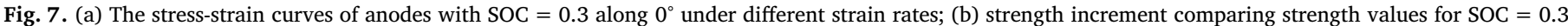
with those for SOC $=0$. Electrolyte effect on the mechanical properties of (c) anode and (d) cathode.
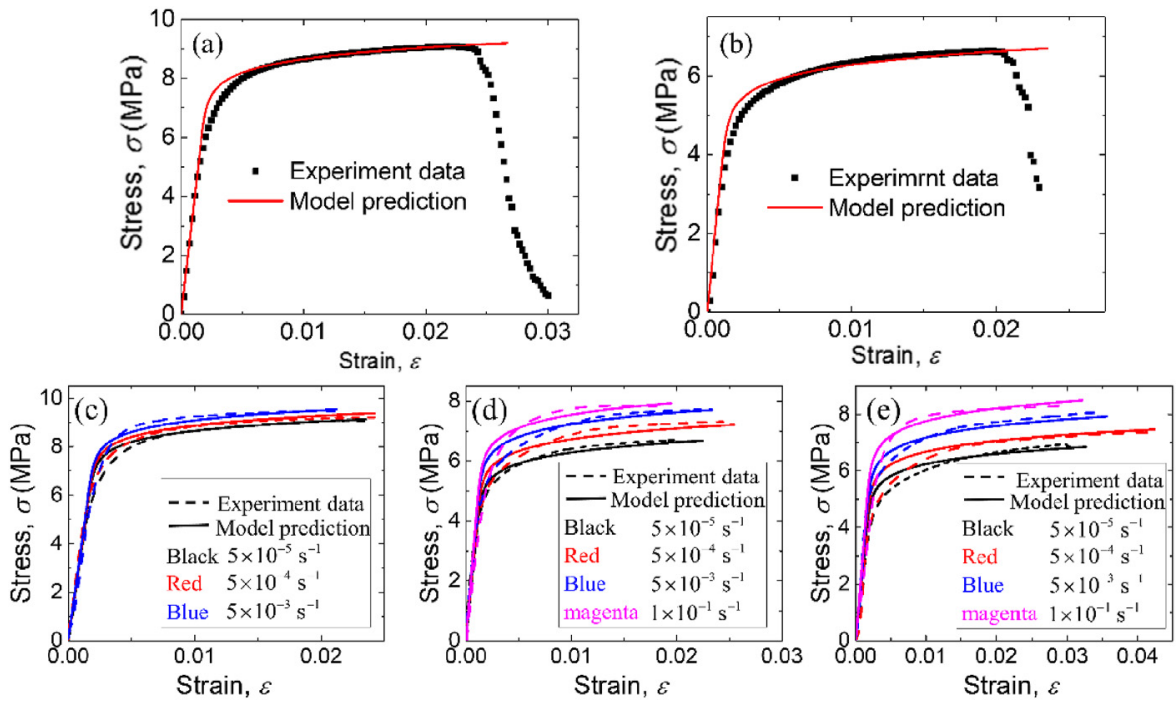

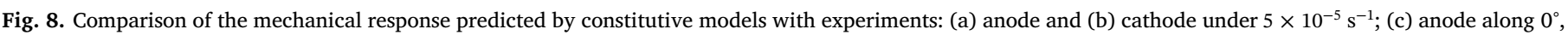
(d) cathode along $0^{\circ}$ and (e) cathode along $90^{\circ}$ with strain rates.

$\varepsilon=\frac{\sigma}{E\left(1+C \ln \frac{\dot{\varepsilon}}{\varepsilon_{0}}\right)}+0.016\left(\frac{\sigma}{9\left(1+C \ln \frac{\dot{\varepsilon}}{\varepsilon_{0}}\right)}\right)^{18.77}$

where the value $\mathrm{C}=0.11$ determined by fitting with the modified expression, $\varepsilon_{0}=5 \times 10^{-5}$, is the reference strain rate, and the model prediction shown in Fig. $8 \mathrm{c}$ are highly consistence in experiment data at different strain rate.

Cathode, whose anisotropic properties mainly reflected in effective modulus and failure strain, an elastic anisotropic model is established as below:

$$
\left\{\begin{array}{l}
\varepsilon_{1} \\
\varepsilon_{2} \\
\gamma
\end{array}\right\}=\left[\begin{array}{ccc}
1 / E_{1} & -v_{12} / E_{1} & 0 \\
-v_{12} / E_{1} & 1 / E_{2} & 0 \\
0 & 0 & 1 / G_{12}
\end{array}\right]\left\{\begin{array}{l}
\sigma_{11} \\
\sigma_{22} \\
\tau_{12}
\end{array}\right\}
$$

where $\quad E_{1}=E_{0}=3620 \mathrm{MPa}, \quad E_{2}=E_{90}=2500 \mathrm{MPa}, \quad v_{12}=0.3 \mathrm{and}$ $G_{12}=1200 \mathrm{MPa}$ obtained from experiment and numerical simulation. The elastic-plastic model can be described as:

$\varepsilon=\frac{\sigma}{E}+0.027\left(\frac{\sigma}{6.8}\right)^{14.95}$

This model can describe the plastic properties well for cathode with strain rate of $5 \times 10^{-5}$, i.e. model prediction along $0^{\circ}$ shown in Fig. 8 b. Furthermore, strain rate effect is included, and the model can be modified as below: 
$\varepsilon=\frac{\sigma}{E\left(1+0.032 \ln \frac{\dot{\varepsilon}}{\varepsilon_{0}}\right)}+0.027\left(\frac{\sigma}{6.8\left(1+0.032 \ln \frac{\dot{\varepsilon}}{\varepsilon_{0}}\right)}\right)^{14.95}$

Similarly, $\varepsilon_{0}=5 \times 10^{-5}$, and the results are shown in Fig. $8 \mathrm{~d}$ and e. The model can well express the anisotropic properties in elastic stage and the strain rate effect. Eqs. (1)-(5) may be easily embedded in current mature computation platforms (e.g. ABAQUS, LS-DYNA) to provide mechanical safety analysis of LIB.

\section{Concluding remarks}

Anode and cathode are key components of LIBs in terms of mechanical integrity, and thus their mechanical properties were thoroughly investigated using macroscopic mechanics and microscopic characterization methods. Tensile tests were carried out for electrode materials with different SOC values from various directions under different stain rates varying from $5 \times 10^{-5}$ to $0.1 \mathrm{~s}^{-1}$. Strong anisotropy was observed in cathode while highly strain-rate dependency existed for both anode and cathode materials in all directions. The properties of anode changed with SOC status while those of cathode did not. Also, tests in different electrolyte conditions revealed weakening effects of the electrolyte were found. Besides, coupling effects of strain rate and SOC were studied, and results showed that strain rate effects were different for materials with different SOC values. SEM technology was utilized to depict the microscopic characteristics for surfaces and crosssections of electrode materials and assist mechanical analysis. Mechanical behavior of sandwiched anode and cathode materials would be influenced not only by each layer but also their interfacial adhesion force, and their failure modes changed with strain rate by SEM images. Subsequently, constitutive models were established for both anode and cathode materials considering strain rate effects and anisotropy. The models developed here are helpful to build the detailed model of lithium-ion battery, which can quantitatively predict the mechanical responses as well as capture the stress state of inner components. Results may shed light on the comprehensive understanding of mechanical behavior of the anode and cathode and facilitate future study in mechanical integrity of LIBs.

\section{Acknowledgement}

LW and SY would like to thank the financial support from The National Key Research and Development Program of China (2017YFB0103703), Opening project of State Key Laboratory of Explosion Science and Technology (Beijing Institute of Technology) (KFJJ17-13M), Research Project of the State Key Laboratory of Vehicle NVH and Safety Technology under Grant NVHSKL-201610. CZ would like to thank the support of National Nature Science Foundation of China (51706187).

\section{References}

[1] P. Peng, F.M. Jiang, Int. J. Heat Mass Tran. 103 (2016) 1008-1016.

[2] J.B. Goodenough, Y. Kim, Chem. Mater. 22 (2010) 587-603.

[3] B. Scrosati, J. Garche, J. Power Sources 195 (2010) 2419-2430.

[4] J.G. Kim, B. Son, S. Mukherjee, N. Schuppert, A. Bates, O. Kwon, M.J. Choi, H.Y. Chung, S. Park, J. Power Sources 282 (2015) 299-322.

[5] P. Ping, Q.S. Wang, P.F. Huang, J.H. Sun, C.H. Chen, Appl. Energy 129 (2014) 261-273.

[6] A. Samba, N. Omar, H. Gualous, O. Capron, P. Van den Bossche, J. Van Mierlo, Electrochim. Acta 147 (2014) 319-329.

[7] M. Chen, D. Zhou, X. Chen, W. Zhang, J. Liu, R. Yuen, J. Wang, J. Therm. Anal. Calorim. 122 (2015) 755-763.

[8] Q. Sun, L. Jiang, L. Gong, J.H. Sun, J. Hazard Mater. 314 (2016) 230-236.

[9] C.-Y. Jhu, Y.-W. Wang, C.-M. Shu, J.-C. Chang, H.-C. Wu, J. Hazard Mater. 192 (2011) 99-107.

[10] P. Ribiere, S. Grugeon, M. Morcrette, S. Boyanov, S. Laruelle, G. Marlair, Energy Environ. Sci. 5 (2012) 5271-5280.

[11] N. Balke, S. Jesse, A.N. Morozovska, E. Eliseev, D.W. Chung, Y. Kim, L. Adamczyk, R.E. Garcia, N. Dudney, S.V. Kalinin, Nat. Nanotechnol. 5 (2010) 749-754.

[12] A. Mukhopadhyay, B.W. Sheldon, Prog. Mater. Sci. 63 (2014) 58-116.

[13] Y. Li, K. Zhang, B. Zheng, X. Zhang, Q. Wang, J. Appl. Phys. 117 (2015).

[14] C. Bonatti, D. Mohr, Mater. Sci. Eng. A 654 (2016) 329-343.

[15] T. Danner, G. Zhu, A.F. Hofmann, A. Latz, Electrochim. Acta 184 (2015) 124-133.

[16] C.V. Di Leo, E. Rejovitzky, L. Anand, Int. J. Solid Struct. 67-68 (2015) 283-296.

[17] Y. Xie, J. Li, C. Yuan, J. Power Sources 248 (2014) 172-179.

[18] R. Kuruba, M.K. Datta, K. Damodaran, P.H. Jampani, B. Gattu, P.P. Patel, P.M. Shanthi, S. Damle, P.N. Kumta, J. Power Sources 298 (2015) 331-340.

[19] D.-E. Yoon, C. Hwang, N.-R. Kang, U. Lee, D. Ahn, J.-Y. Kim, H.-K. Song, ACS Appl. Mater. Interfaces 8 (2016) 4042-4047.

[20] D. Shi, X. Xiao, X. Huang, H. Kia, J. Power Sources 196 (2011) 8129-8139.

[21] A. Sheidaei, X.R. Xiao, X.S. Huang, J. Hitt, J. Power Sources 196 (2011) 8728-8734.

[22] C.M. Costa, M.M. Silva, S. Lanceros-Mendez, RSC Adv. 3 (2013) 11404-11417.

[23] J. Nunes-Pereira, C.M. Costa, R. Leones, M.M. Silva, S. Lanceros-Méndez, Solid State Ionics 249-250 (2013) 63-71.

[24] I. Avdeev, M. Martinsen, A. Francis, J. Mater. Eng. Perform. 23 (2014) 315-325.

[25] J. Cannarella, X.Y. Liu, C.Z. Leng, P.D. Sinko, G.Y. Gor, C.B. Arnold, J. Electrochem. Soc. 161 (2014) F3117-F3122.

[26] K.Y. Oh, J.B. Siegel, L. Secondo, S.U. Kim, N.A. Samad, J.W. Qin, D. Anderson, K. Garikipati, A. Knobloch, B.I. Epureanu, C.W. Monroe, A. Stefanopoulou, J. Power Sources 267 (2014) 197-202.

[27] S. Yan, X. Xiao, X. Huang, X. Li, Y. Qi, Polymer (United Kingdom) 55 (2014) 6282-6292.

[28] G.Y. Gor, J. Cannarella, C.Z. Leng, A. Vishnyakov, C.B. Arnold, J. Power Sources 294 (2015) 167-172.

[29] J.C. Chen, Y.D. Yan, T. Sun, Y. Qi, X.D. Li, RSC Adv. 4 (2014) 14904-14914.

[30] A. Sheidaei, X. Xiao, X. Huang, J. Wang, SAE 2011 World Congress and Exhibition, 2011.

[31] J. Xu, L. Wang, J. Guan, S. Yin, Mater. Des. 95 (2016) 319-328.

[32] W.-J. Lai, M.Y. Ali, J. Pan, J. Power Sources 248 (2014) 789-808.

[33] E. Sahraei, M. Kahn, J. Meier, T. Wierzbicki, RSC Adv. 5 (2015) 80369-80380.

[34] J. Xu, B. Liu, D. Hu, Sci. Rep.-UK 6 (2016).

[35] Z. Ma, H. Wu, Y. Wang, Y. Pan, C. Lu, Int. J. Plast. 88 (2017) 188-203.

[36] C. Zhang, J. Xu, L. Cao, Z. Wu, S. Santhanagopalan, J. Power Sources 357 (2017) 126-137.

[37] H. Yang, M. Citrin, X. Xia, S. Nieh, J. Greer, ECS Meeting, Washington State Convention Center, 2018.

[38] Y. Bai, X. Wang, X. Zhang, H. Shu, X. Yang, B. Hu, Q. Wei, H. Wu, Y. Song, Electrochim. Acta 109 (2013) 355-364.

[39] Y. Arachi, H. Kobayashi, S. Emura, Y. Nakata, M. Tanaka, T. Asai, H. Sakaebe, K. Tatsumi, H. Kageyama, Solid State Ionics 176 (2005) 895-903.

[40] G.G. Botte, Electrochim. Acta 50 (2005) 5647-5658.

[41] J. Swiatowska-Mrowiecka, V. Maurice, S. Zanna, L. Klein, E. Briand, I. Vickridge, P. Marcus, J. Power Sources 170 (2007) 160-172.

[42] L. Brassart, Z. Suo, J. Mech. Phys. Solids 61 (2013) 61-77. 